\author{
Andreas Christ \\ Christian A. Arranto \\ Christian Schindler \\ Theresia Klima \\ Patrick R. Hunziker \\ Martin Siegemund \\ Stephan C. Marsch \\ Urs Eriksson \\ Christian Mueller
}

\section{Incidence, risk factors, and outcome of aspiration pneumonitis in ICU overdose patients}

Received: 23 August 2005

Accepted: 9 June 2006

Published online: 7 July 2006

(C) Springer-Verlag 2006

\author{
A. Christ - C. A. Arranto - T. Klima . \\ U. Eriksson · C. Mueller ( $)$ \\ University of Basel, University Hospital, \\ Department of Internal Medicine, \\ Petersgraben 4, 4031 Basel, Switzerland \\ e-mail: chmueller@uhbs.ch \\ Tel.: +41-61-2652525 \\ Fax: +41-61-2655353 \\ C. Schindler \\ University of Basel, University Hospital, \\ Institute for Social and Preventive \\ Medicine, \\ Petersgraben 4, 4031 Basel, Switzerland \\ P. R. Hunziker · M. Siegemund • \\ S. C. Marsch \\ University of Basel, University Hospital, \\ Division of Intensive Care, Department of \\ Internal Medicine, \\ Petersgraben 4, 4031 Basel, Switzerland
}

\begin{abstract}
Objective: To assess the incidence and outcome of clinically significant aspiration pneumonitis in intensive care unit (ICU) overdose patients and to identify its predisposing factors. Design: Retrospective cohort study. Setting: Medical ICU of an academic tertiary care hospital. Patients: A total of 273 consecutive overdose admissions. Measurements and results: Clinically significant aspiration pneumonitis was defined as the occurrence of respiratory dysfunction in a patient with a localised infiltrate on chest X-ray within $72 \mathrm{~h}$ of admission. In our cohort we identified 47 patients $(17 \%)$ with aspiration pneumonitis. Importantly, aspiration pneumonitis was associated with a higher incidence of cardiac arrest (6.4 vs $0.9 \% ; p=0.037)$ and an increased duration of both ICU stay and overall hospital stay [respectively: median 1 (interquartile range $1-3$ ) vs
\end{abstract}

$1(1-2), p=0.025 ;$ and median $2(1-7)$ vs $1(1-3), p<0.001]$. In multivariate logistic regression analysis, Glasgow Coma Scale (GCS) score [odds ratio (OR) for each point of GCS 0.8 ; 95\% confidence interval (CI) 0.7-0.9; $p=0.001]$, ingestion of opiates (OR 4.5 ; 95\% CI 1.7-11.6; $p=0.002)$, and white blood cell count (WBC) (OR for each increase in WBC of $10^{9} / 1$ $1.05 ; 95 \%$ CI $1.0-1.19 ; p=0.049$ ) were identified as independent risk factors. Conclusions: Clinically relevant aspiration pneumonitis is a frequent complication in overdose patients admitted to the ICU. Moreover, aspiration pneumonitis is associated with a higher incidence of cardiac arrest and increased ICU and total in-hospital stay.

Keywords Aspiration · Pneumonitis . Overdose $\cdot$ Intensive care

\section{Introduction}

Drug overdose is an important and increasingly frequent cause of admission to the ICU, and accounts for significant morbidity and mortality [1, 2, 3]. In 2001, approximately 15.9 million Americans had used an illicit drug at least once in the month prior to being interviewed [2]. Moreover, hospital use due to overdose has increased. Mortality rate was $3.9 \%$ in a recent report of patients with acute poisoning admitted to the emergency department [3].

Aspiration pneumonitis (AP) is a common complication of drug overdose $[2,4,5]$. AP results from the aspiration of gastric contents, which causes acute chemical inflammation and shows a broad spectrum of clinical outcomes, ranging from asymptomatic and self-limiting episodes to the development of severe pneumonitis with rapid progression to acute respiratory distress syndrome $[6,7]$. Bacterial colonisation leading to aspiration pneumonia and sepsis are uncommon sequelae, although in severe cases they may occur.

There are only few studies of aspiration in overdose patients, and most focus on the risk of aspiration with gastric emptying [5]. In addition, overlap between the clinical entities of AP and aspiration pneumonia complicates epidemiological and clinical studies of AP. Therefore, 
little is known about the incidence and risk factors of AP [6]. This study sought to determine the frequency and outcome of clinically significant AP in an ICU population of overdose patients, and to identify its predisposing factors.

\section{Materials and methods}

Setting and study population

We performed a retrospective observational study in consecutive overdose patients admitted to the medical ICU of the University Hospital Basel, Switzerland, within a 3-year period. AP was recorded as the primary endpoint. Management in general included repetitive administrations of activated charcoal [8]. Gastric emptying was not performed in any of the patients. Prospective data collection was approved by the local ethics committee and was in accordance with the principles of the Declaration of Helsinki.

\section{Endpoints}

Clinically significant AP was defined as the occurrence of respiratory dysfunction in a patient with a localised pulmonary infiltrate on chest X-ray [5]. Chest X-ray was routinely performed in this cohort. All patients with an obvious or at least suspected local infiltrate were identified as potential cases and therefore reviewed for significant respiratory dysfunction. Respiratory dysfunction was defined as at least one of the following: invasive or non-invasive mechanical ventilation for hypoxaemia or hypercapnia, supplemental oxygen $>4 \mathrm{l} / \mathrm{min}$; respiratory rate $>20 / \mathrm{min}$, arterial oxygen saturation $<92 \%, \mathrm{PaO}_{2}<10 \mathrm{kPa}$ within the first $72 \mathrm{~h}$ after admission.

We identified ingested drugs using ambulance charts, history, urine screening and blood analysis. Patients were contacted 6 months after discharge by telephone.

\section{Statistical analysis}

Results are expressed as means \pm SD for continuous normally distributed variables. For non-parametric data, medians with interquartile ranges are used. To describe baseline characteristics, comparisons were made using the $t$-test, Mann-Whitney $U$-test, Fisher's exact test and chi-square test as appropriate. Univariate and multivariate analyses were conducted using logistic regression. The following variables were entered in multivariate analysis: Glasgow Coma Scale (GCS) score on admission, opiate ingestion, APACHE II, heart rate on admittance, serum sodium, serum potassium, white blood cell count (WBC). Risk-adjusted mortality rates (observed/expected) were compared for patients with and without AP, using the diagnostic category weight of drug overdose for the expected rates $[9,10]$. Tests were two-sided, and a $p$ value of less than 0.05 was considered to indicate statistical significance. Statistical analyses were performed using SPSS/PC (version 12.0, SPSS Inc.).

\section{Results}

Between January 1998 and August 2001, of a total of 3499 ICU patients 273 were admitted for intoxication. Using the predefined criteria, 61 patients $(22 \%)$ showed a localised infiltrate on chest X-ray. Of these, $47(17 \%)$ additionally had respiratory dysfunction. Thus, a total of 47 patients (17\%; 95\% CI 13-22\%) met the criteria for clinical relevant AP. As shown in Table 1, there were important differences among baseline characteristics in patients with and without AP. Values are given as median (interquartile range), if not otherwise indicated.

The GCS score on admittance of patients with AP was 7 (3-9), compared with 14 (7-15) in patients without AP. Sixty-one per cent of all patients (167 of 273) had a GCS score of 14 or less, $26 \%$ (70 of 273) a GCS score of less than 8. APACHE II score was significantly higher in patients with AP than in patients without (Table 1).

Mixed drug intoxication was present in most patients. Ingestion of opiates was identified in $38 \%$ of patients with AP and only in $12 \%$ of those without. Other substances were ingested in similar frequency by patients with and without AP.

\section{Outcome}

A total of 7 patients $(2.6 \%)$ died during the hospitalisation, with similar risk-adjusted mortality rates in patients with and without AP. The high O/E ratios are caused by the chosen diagnostic category weight of drug overdose, which leads to relatively low expected mortality rates.

The length of stay was significantly longer in patients with AP than in patients without AP, both in the ICU and overall in hospital. Three patients with AP suffered a cardiac arrest $(6.4 \%)$ while in the ICU, against two patients without AP $(0.9 \% ; p=0.037)$. There were no deaths during the 6-month follow-up period.

\section{Risk factor analyses for aspiration pneumonitis}

Several predictors of AP could be identified in univariate logistic regression (Table 2). Of these, GCS score on admittance, opiate ingestion, and WBC remained statistically significant independent predictors of AP in multivariate logistic regression. 
Table 1 Baseline characteristics and outcome

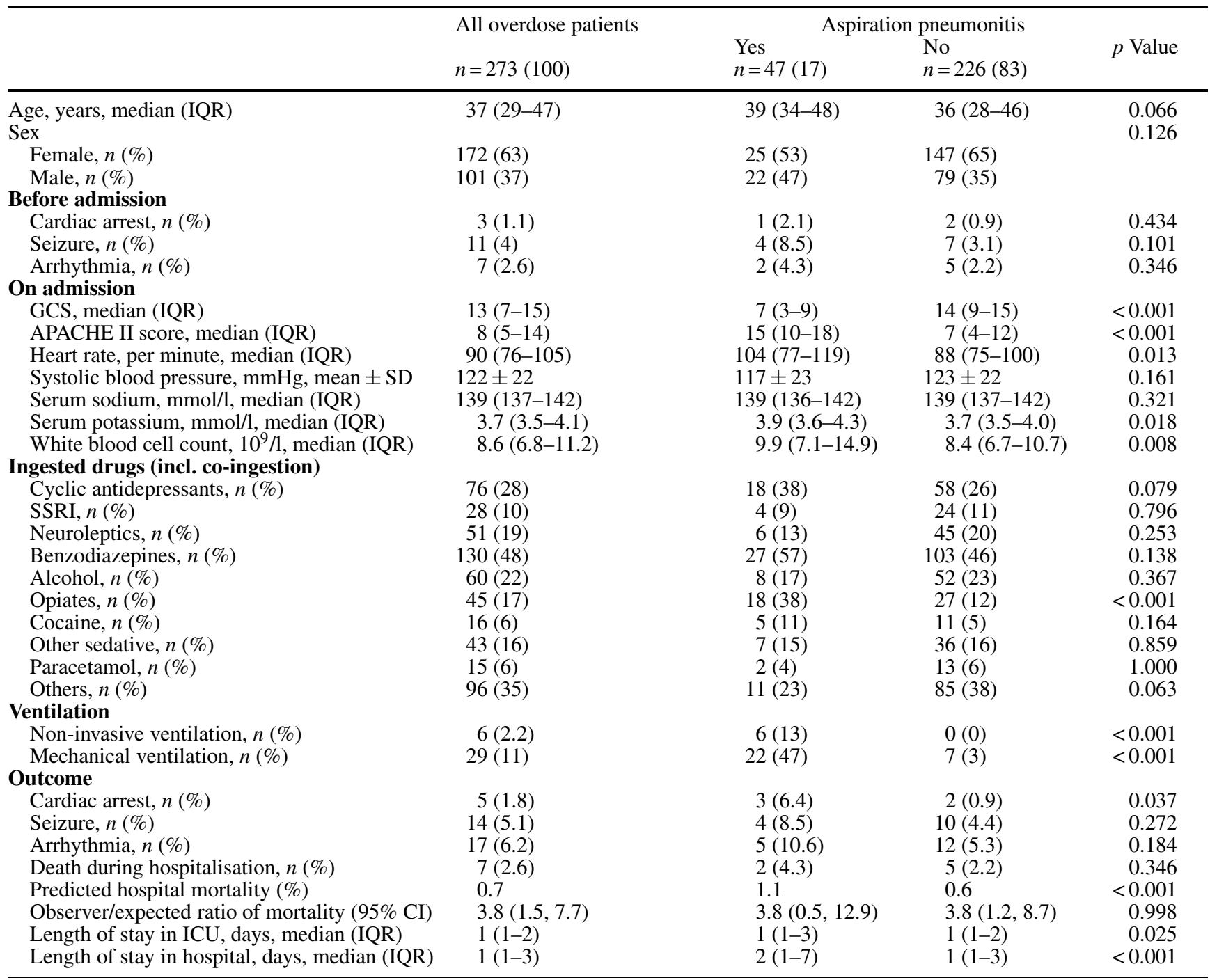

GCS, Glasgow Coma Scale score [14]; APACHE II, Acute Physiologic and Chronic Health Evaluation II [9]

Table 2 Predictors of aspiration pneumonitis in univariate and multivariate analyses

\begin{tabular}{lllc}
\hline Variable & Odds ratio & $\begin{array}{l}95 \% \text { Confidence } \\
\text { interval }\end{array}$ & $p$ Value \\
\hline Univariate analysis & & & \\
$\quad$ GCS on admittance (continuous) & 0.81 & $0.75-0.87$ & $<0.001$ \\
$\quad$ Opiate ingestion & 4.58 & $2.24-9.33$ & $<0.001$ \\
$\quad$ APACHE II (continuous) & 1.17 & $1.10-1.24$ & $<0.001$ \\
$\quad$ Heart rate on admittance (per minute) & 1.02 & $1.00-1.03$ & 0.027 \\
$\quad$ continuous) & 0.94 & $0.88-1.00$ & 0.039 \\
$\quad$ Serum sodium (mmol/l) (continuous) & 1.81 & $1.17-2.81$ & 0.008 \\
$\quad$ Serum potassium (mmol/l) (continuous) & 1.11 & $1.04-1.19$ & 0.002 \\
$\quad$ White blood cell count (10 $/ 1$ ) (continuous) & & $0.75-0.93$ & 0.001 \\
Multivariate analysis & 0.83 & $1.74-11.60$ & 0.002 \\
$\quad$ GCS on admittance (continuous) & 4.50 & $1.00-1.19$ & 0.049 \\
$\quad$ Opiate ingestion & 1.05 & & \\
$\quad$ White blood cell count $\left(10^{9} / 1\right)$ (continuous) & & & \\
\hline
\end{tabular}

Continuous variables: odds ratios (OR) for each increase of one unit of the respective variable. For instance, a patient with a GCS of 15 on admittance has an OR of 0.81 for aspiration pneumonitis compared with a patient with a GCS of 14 


\section{Discussion}

This study shows that clinically relevant $\mathrm{AP}$ is a frequent complication in an unselected cohort of consecutive overdose patients admitted to the ICU. In addition, AP was associated with an increased length of stay in the ICU and in hospital, and a higher incidence of cardiac arrest. GCS on admittance, ingestion of opiates, and WBC were identified as readily definable independent risk factors for AP.

The incidence of AP in our population is within the wide range found in previous studies. Differences in the types of drugs abused, ICU admission strategies, incidence of risk factors and definition of clinical significant AP seem to account for most of the difference in incidence. Isbister and colleagues reported a frequency of AP of $11 \%$ in unselected overdose patients admitted to the ICU in Australia [5]. In a study of the relation between GCS score and the incidence of AP, the rate of AP in overdose patients admitted to the ICU was $29 \%$ [11]. A GCS score of 14 or less was present in $85 \%$ of patients in that study, compared with $61 \%$ in our study. Since deeper unconsciousness as indicated by a lower GCS score is associated with a higher risk of aspiration, the difference in GCS score may well be responsible for the different incidence of AP in the two studies. Also, a high incidence of AP of $28 \%$ was found in a Finnish population [12]. In that cohort, $68 \%$ of patients were unconscious, with unconsciousness defined as a GCS score of 3-7. In contrast, only $26 \%$ of our patients had a GCS score of 3-7. Again, the difference in the level of consciousness between the two populations might account for the different frequency of AP.

In our study, AP was associated with significantly increased morbidity: patients with AP had longer LOS both in the ICU and in hospital. This finding is supported by recent data from Australia [5]. In addition, our study shows that patients with AP have a higher rate of cardiac arrest. Mortality overall was low in our cohort. Therefore we had limited statistical power to investigate the impact of AP on mortality. In our cohort, risk-adjusted mortality rates showed no differences between patients with AP and without. Isbister and colleagues reported significantly greater overall mortality in patients with AP than in patients without AP in a very large overdose population [5].

Given the clinically significant increased morbidity in patients with AP, it is mandatory to identify easily and rapidly assessable risk factors for AP. Our study clearly shows the important role of the GCS score, the ingestion of opiates and an increased WBC as independent predictors for AP. All three risk factors are easily available during the initial assessment in the ICU and therefore very useful in clinical practice. Differences in baseline patient characteristics, study setting, and definition of clinically significant AP may account for the difference in independent predictors found in previous studies [5, 12]. Additional prospective studies are necessary to validate the risk factors found in our study.

Several limitations apply to our study: firstly, our definition of AP may have included some patients with early aspiration pneumonia. Although the identification of secondary bacterial colonisation and infection is difficult, the rate of this complication was low, at most, as we included only patients with infiltration and respiratory dysfunction within $72 \mathrm{~h}$ after the admission. Infectious complications in general become clinically apparent only after several days of pulmonary aspiration. Secondly, it is possible that in our study some patients had other pulmonary complications of opiate intoxication rather than AP [2]. Particularly heroin can cause pulmonary oedema. Using a localised infiltrate on chest X-ray as inclusion criterion for AP should have excluded most cases of pulmonary oedema, since heroin-induced pulmonary oedema manifests typically as widespread, bilateral airspace consolidation on chest X-ray [13].

In conclusion, relevant $\mathrm{AP}$ is a frequent complication of overdose patients admitted to the ICU. Three readily assessable variables identify patients at risk for AP. This is clinically important, as AP is associated with increased morbidity.

\section{References}

1. Henderson A, Wright M, Pond SM (1993) Experience with 732 acute overdose patients admitted to an intensive care unit over six years. Med J Aust 158:28-30

2. Wilson KC, Saukkonen JJ (2004) Acute respiratory failure from abused substances. J Intensive Care Med 19:183-193

3. Satar S, Seydaoglu G (2005) Analysis of acute adult poisoning in a 6-year period and factors affecting the hospital stay. Adv Ther 22:137-147
4. Roy TM, Ossorio MA, Cipolla LM, Fields CL, Snider HL, Anderson WH (1989) Pulmonary complications after tricyclic antidepressant overdose. Chest 96:852-856

5. Isbister GK, Downes F, Sibbritt D, Dawson AH, Whyte IM (2004) Aspiration pneumonitis in an overdose population: frequency, predictors, and outcomes. Crit Care Med 32:88-93

6. Marik PE (2001) Aspiration pneumonitis and aspiration pneumonia. $\mathrm{N}$ Engl $\mathrm{J}$ Med 344:665-671
7. Lu Y, Song Z, Zhou X, Huang S, Zhu D, Yang CBX, Sun B, Spragg R (2004) A 12-month clinical survey of incidence and outcome of acute respiratory distress syndrome in Shanghai intensive care units. Intensive Care Med 30:2197-2203

8. Bond GR (2002) The role of activated charcoal and gastric emptying in gastrointestinal decontamination: a state-of-the-art review. Ann Emerg Med 39:273-286 
9. Knaus WA, Draper EA, Wagner DP, Zimmerman JE (1985) APACHE II: a severity of disease classification system. Crit Care Med 13:818-829

10. Hosmer DW, Lemeshow S (1995) Confidence interval estimates of an index of quality performance based on logistic regression models. Stat Med 14:2161-2172
11. Adnet F, Baud F (1996) Relation between Glasgow Coma Scale and aspiration pneumonia. Lancet 348:123-124

12. Liisanantti J, Kaukoranta P, Martikainen M, Ala-Kokko T (2003) Aspiration pneumonia following severe self-poisoning. Resuscitation 56:49-53
13. Gluecker T, Capasso P, Schnyder P, Gudinchet F, Schaller MD, Revelly JP, Chiolero R, Vock P, Wicky S (1999) Clinical and radiologic features of pulmonary edema. Radiographics 19:1507-1531; discussion 1532-1533

14. Chan B, Gaudry P, Grattan-Smith TM, McNeil R (1993) The use of Glasgow Coma Scale in poisoning. J Emerg Med 11:579-582 